\title{
Decompressive Craniectomy in Posterior Fossa Ischemic Stroke
}

\author{
Luciano Santana-Cabrera, Guillermo Pérez-Acosta, Cristina Rodríguez-Escot, Rosa Lorenzo-Torrent, \\ Manuel Sánchez-Palacios
}

Intensive Care Unit, Insular Universitary Hospital of Gran Canaria, Las Palmas of Gran Canaria, Spain.

Email: lsancabx@gobiernodecanarias.org

Received March 11 ${ }^{\text {th }}, 2012$; revised April 24 ${ }^{\text {th }}, 2012$; accepted May 29 $9^{\text {th }}, 2012$

\begin{abstract}
Ischemic damage produced in the posterior cerebral territory causes significant morbidity and urgently must be considered if the patient need a surgical attitude. Surgical decompression by suboccipital craniectomy seams to be effective to treat secondary edema due to cerebellar damage or in posterior fossa, when medical treatment is not able to control side effects. We report a clinical case of a patient with a subacute ischemic infarction in the vertebro-basilar territory, with perilesional edema, and a posterior fossa decompressive craniectomy (DC) was carried out.
\end{abstract}

Keywords: Decompressive Craniectomy; Posterior Fossa; Suboccipital Craniectomy; Vertebrobasilar Insufficiency

\section{Introduction}

Ischemic stroke in the posterior cerebral artery (PCA) territory is a frequent pathology, and the most common causes are usually the cardioembolic and the vertebral or basilar artery dissection. The initial symptom is usually visual field defects, but may eventually lead to sensory and motor deficits. When an alteration of the level of consciousness appears, is very likely already multi-infarct exist or has affectation of a large territory of posterior circulation [1].

It is in this context that we must consider whether the patient is subsidiary of a procedure like DC [2]. In this article in this paper we discuss the role of craniectomy in the posterior fossa ischemic stroke.

\section{Clinical Case}

We report a clinical case of a 68 years old male patient, with hypertension and smoker, that suffered a syncope with momentary loss of consciousness, and when arriving to the emergency department presented neurological deterioration with facial paralysis, pupillary anisocoria, hypoesthesia in the left upper limb, left homonymous hemianopsia, dysarthria, bradycardia and apnea, being intubated. A Cranial CT was performed without evidence of hemorrhagic lesions and, with a significant stenosis at the origin of the left vertebral artery. In the cerebral arteriography performed, was objectified the left vertebral artery stenosis, being unable to place a stent, due to the high risk of complete obstruction, being unique. Intrave- nous thrombolysis was initiated without evidence of improvement in his neurological status. At 48 hours of ICU admission, further neurological deterioration, so a cranial CT was performed, showing the presence of a subacute ischemic infarction in the vertebro-basilar territory, with perilesional edema, and a posterior fossa DC was carried out. Without neurologic improvement after the DC, the patient was discharged from the ICU, 15 days after admission, with a GCS of 5 points and a CT scan which demonstrated, ischemic lesions in the left posterior cerebral artery territory and both cerebellar hemispheres with hemorrhagic transformation (Figures 1(a) and (b)).

\section{Discussion}

Ischemic damage produced in the posterior cerebral territory by non-traumatic causes such as atheroembolic, the rupture of an aneurysm, arterio-venous malformation, or vertebral artery dissection, causes significant morbidity and urgently must be considered a surgical attitude. Surgical decompression by suboccipital craniectomy seams to be effective to treat secondary edema due to cerebellar damage or in posterior fossa, when medical treatment is not able to control side effects.

We tend to use decompressive suboccipital craniectomy with temporary ventriculostomy instead of simply ventriculostomy because of the potential complications of this one, as the possibility of cerebellum herniationthrough the tentorium and cerebral parenchymal compression [3]. Another relevant complication is the post- 


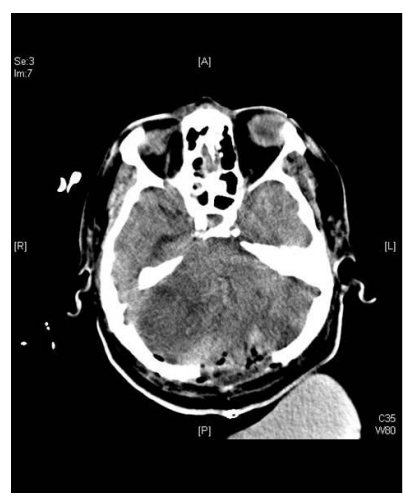

(a)

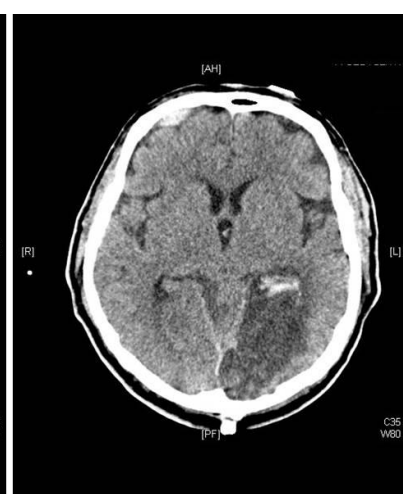

(b)

Figure 1. CT where ischemic damage is visualized at the level of brainstem (a) and hemorrhagic transformation of ischemic infarction in the territory of the posterior communicating artery (b).

operative infection, as the permanence of the drainage catheters for longer than 6 days, increases the risk of infection, which increases morbi-mortality to a patology which itself, has a poor prognosis.

The fundamental criteria for the decision of surgical treatment of posterior fossa decompression, with universal acceptance, are the clinical and radiological signs of brainstem compression [4].

The main problem is that not only the clinical situation is useful to make a decision of the management in these patients, because they can suffer a neurological deterioration despite being neurologically stable, and here is where radiological images play an important role.

However, the compression of posterior fossa structures not only depends on the edema, it is also important the relationship between these structures and the available space in this cavity, with significant variability. Thus, ischemic processes and edema, are much better tolerated

in the presence of atrophy and worse in those cases of narrower fossas.

\section{Conclusion}

In conclusion, the decision to perform a posterior fossa craniectomy must be evaluated as son as possible, according to clinical and radiological evolution, although the value of all different types of treatment and prognosis of patient is unclear, and needs more prospective studies [5].

\section{REFERENCES}

[1] E. Kumral, G. Bayulkem, C. Atac and Y. Alper, "Spectrum of Posterior Cerebral Artery Surface Infartcs Territory,” European Journal of Neurology, Vol. 11, 2004, pp. 237-246. doi:10.1046/j.1468-1331.2003.00750.x

[2] H. Kudo, T. Kawaguchi, H. Minami, K. Kuwamura, M. Miyata and E. Kohmura, "Controversy of Surgical Treatment for Severe Cerebellar Infarction,” Journal of Stroke and Cerebrovascular Diseases, Vol. 16, No. 6, 2007, pp. 259-262. doi:10.1016/j.jstrokecerebrovasdis.2007.09.001

[3] G. Lungu, J. Mortada and B. Stilhart, "Surgical Treatment of Cerebellar Infarction: Five Case Studies," Neurochirurgie, Vol. 56, 2010, pp. 59-62.

doi:10.1016/j.neuchi.2009.11.011

[4] P. P. Tsitsopoulos, L. Tobieson, P. Enblad and N. Marklund, "Following Surgical Outcome Clinical Treatment for Bilateral Cerebellar Infarction,” Acta Neurologica Scandinavica, Vol. 123, 2011, pp. 345-351. doi:10.1111/j.1600-0404.2010.01404.x

[5] E. Jüttler, S. Schweickert, P. A. Ringleb, H. B. Huttner, M. Köhrmann and A. Aschoff, "Long-Term Outcome after Surgical Treatment for Space-Occupying Cerebellar Infarction: Experience in 56 Patients,” Stroke, Vol. 40, 2009, pp. 3060-3066. doi:10.1161/STROKEAHA.109.550913 